\title{
APPROXIMATE CONTROLLABILITY AND ITS WELL-POSEDNESS FOR THE SEMILINEAR REACTION-DIFFUSION EQUATION WITH INTERNAL LUMPED CONTROLS
}

\author{
Alexander Khapalov ${ }^{1}$
}

\begin{abstract}
We consider the one dimensional semilinear reaction-diffusion equation, governed in $\Omega=$ $(0,1)$ by controls, supported on any subinterval of $(0,1)$, which are the functions of time only. Using an asymptotic approach that we have previously introduced in [9], we show that such a system is approximately controllable at any time in both $L^{2}(0,1)$ and $C_{0}[0,1]$, provided the nonlinear term $f=f(x, t, u)$ grows at infinity no faster than certain power of $\log |u|$. The latter depends on the regularity and structure of $f(x, t, u)$ in $x$ and $t$ and the choice of the space for controllability. We also show that our results are well-posed in terms of the "actual steering" of the system at hand, even in the case when it admits non-unique solutions.
\end{abstract}

Résumé. On étudie l'équation de la chaleur semi-linéaire sur l'intervalle $(0,1)$ avec des contrôles internes sur un sous-intervalle et qui ne sont que des fonctions du temps. Utilisant une approche asymptotique que nous avons précédemment introduite dans [9], on montre la contrôlabilité approchée pour tout temps à la fois dans $L^{2}(0,1)$ et dans $C_{0}[0,1]$ si le terme non linéaire $f=f(x, t, u)$ ne croit pas plus vite qu'une certaine puissance de log $|u|$. Celle-ci dépend de la régularité et de la structure de $f(x, t, u)$ par rapport à $x$ et $t$ et du choix de l'espace pour la contrôlabilité. On montre aussi que nos résultats permettent de piloter le système considéré, même dans le cas où l'on n'a pas unicité des solutions.

AMS Subject Classification. 9335.

Received April 22, 1997. Revised June 24, 1998.

\section{INTRODUCTION}

\subsection{Problem background and literature}

In the past few years considerable attention has been given to the study of global approximate controllability of the following initial-boundary value problem for the semilinear heat equation:

$$
\begin{aligned}
u_{t} & =\Delta u-f(x, t, u, \nabla u)+v(x, t) \chi_{\omega}(x) \text { in } Q_{T}=\Omega \times(0, T), \\
u & =0 \quad \text { in } \Sigma_{T}=\partial \Omega \times T, \quad u(x, 0)=u_{0}(x) \quad \text { in } \Omega,
\end{aligned}
$$

\footnotetext{
Keywords and phrases: The semilinear reaction-diffusion equation, approximate controllability, internal lumped control, multiple solutions.

${ }^{1}$ Department of Pure and Applied Mathematics, Washington State University, Pullman, WA 99164-3113, USA;

e-mail: khapala@delta.math.wsu.edu
}

(c) EDP Sciences, SMAI 1999 
where the term $v(x, t) \chi_{\omega}(x)$ models locally distributed controls supported on $\omega \subset \Omega$ (so that $\chi_{\omega}(x)$ is the characteristic function of $\omega$ ). The question of interest here can be formulated as follows: Given a phase-space $H$ for solutions of $(S)$ and a space $V$ for controls, for what nonlinear terms the range of the solution mapping $V \ni v \rightarrow u(\cdot, T)$ is dense in $H$ ?

Most of the success in this direction was associated with nonlinear terms satisfying the global Lipschitz condition in $u$ with a constant which does not depend on $x$ and $t([3,4,6,7,20,21]$, see also the bibliography therein). This condition, on the one hand, guarantees the well-posedness of the system at hand and, on the other hand, provides the basis for the fixed point argument to solve the approximate controllability problem. The methods of the aforementioned works make use of the variational approach to the issue of controllability [11] and the unique continuation property of solutions to the linear equations from an open subset $\omega \times(0, T)[2,17]$.

\subsection{Problem setting and the method description}

In this article we consider a quite different setup of this problem. Namely, we are concerned with the following one dimensional system:

$$
\begin{aligned}
u_{t} & =u_{x x}-f(x, t, u)+v(t) \chi_{\left(l_{1}, l_{2}\right)}(x) \text { in } Q_{T}=(0,1) \times(0, T), v \in L^{2}(0, T), \\
u(0, t) & =u(1, t)=0, \quad u(x, 0)=u_{0}(x)
\end{aligned}
$$

which is governed by so-called lumped controls $v=v(t)$, with support on a given subinterval $\left(l_{1}, l_{2}\right)$. These controls are the functions of time only, which is motivated by numerous applications. In the technical aspect, this means that one can no longer use the aforementioned unique continuation property.

While for the standard linear heat equation this problem is well-understood by now $[1,5,13,15]$, little is known regarding the semilinear case. Among early works in this area we can mention only [19], dealing with uniformly bounded Lipschitz nonlinearity. For more recent results see [9].

The method, which we use in this article, is quite different from the classical fixed point or implicit function arguments. It is as follows.

Let (1.1) admit a unique solution in $C([0, T], B)$, where $B$ is a Banach space. If $S(t)$ is the semigroup associated with the linear part of (1.1), then:

$$
u(t)=S(t) u_{0}+\int_{0}^{t} S(t-\tau)\left(v \chi_{\left(l_{1}, l_{2}\right)}\right)(\tau) d \tau-\int_{0}^{t} S(t-\tau)(f(\cdot, \cdot, u))(\tau) d \tau .
$$

Assume that the corresponding truncated linear problem (i.e., with $f \equiv 0)$

$$
u_{L t}=u_{L x x}+v(t) \chi_{\left(l_{1}, l_{2}\right)}(x) \text { in } Q_{T}, \quad u_{L}(0, t)=u_{L}(1, t)=0, \quad u_{L}(x, 0)=u_{0}(x)
$$

is approximately controllable in $B$ at any time $T>0$. Then, since its solution has the form of the first two terms on the right in (1.2), for any target state $u_{1} \in B$ and any $t>0$ there is a control $v_{(t)}$ such that

$$
S(T) u_{0}+\int_{0}^{t} S(t-\tau)\left(v_{(t)} \chi_{\left(l_{1}, l_{2}\right)}\right)(\tau) d \tau \rightarrow u_{1} \text { in } B \text { as } t \rightarrow 0 .
$$

In turn (1.2) and (1.4) imply that, if for the solutions $u_{(t)}$ to $(1.1)$ on $(0, t)$, corresponding to $v_{(t)}$, we show that

$$
\left\|\int_{0}^{t} S(t-\tau)\left(f\left(\cdot, \cdot, u_{(t)}\right)\right)(\tau) d \tau\right\|_{B} \rightarrow 0 \text { as } t \rightarrow 0
$$


then $u_{(t)}$ will also converge to $u_{1}$ as $t \rightarrow 0$. Once we managed to show this for a dense set of $u_{1}$ 's in $B$, then we have proven that, given $u_{0}$, any element of $B$ can be approached with an arbitrary accuracy as "quickly" in time as we wish.

Now note that the truncated linear problem (1.3) is time-invariant. Hence, the above constructions apply on any time-interval $\left(t_{0}, T\right)$ with $u\left(\cdot, t_{0}\right)$ in place of $u_{0}$. Hence, if we apply any control $v$, say $v \equiv 0$ on $\left(0, t_{0}\right)$, then, given $u_{0}$, any element of $B$ can be approached with an arbitrary accuracy at some moment $t_{*}$ lying arbitrarily close to $T$. (To do that, select $t_{0}$ "close" to $T$.) Hence, by the continuity properties of (1.1), any element of $B$ can be approached (arbitrarily close) by the trajectories of (1.1) at time $T$. (To show that, apply again the zero control on $\left(t_{0}, T\right)$.) This proves the approximate controllability of (1.1) in $B$ at time $T$.

Remark 1.1. Obviously, if $f=f(x, t, u)$ is uniformly bounded, then (1.5) holds, which immediately gives the result of [19] dealing with bounded $f(x, t, u)$.

Furthermore, since the linear controllability problem is well-understood nowadays, one can select a suitable dense set of targets $u_{1}$ in $B$ without any difficulty. Hence, as (1.5) suggests, the crux of our method is the asymptotic behavior of the nonlinear term with $u$ generated by the controls solving the truncated linear controllability problem. In this respect, our proofs below are based on the asymptotic analysis of the corresponding biorthogonal sequences to $\left\{e^{\lambda_{k} \tau}, k=1, \ldots\right\}$ on $(0, t)$ as $t \rightarrow 0$, where $\lambda_{k}$ 's are the eigenvalues to the Laplace operator.

The disadvantage of this method is that, to ensure (1.5), one has to impose severe assumptions on the growth rate of $f$ in $u$. In that respect, the above-referred results for locally distributed controls provide better growth rates. Accordingly, our goal is to use our method in the situations where the methods of the above-mentioned works do not apply. We distinguish two areas: (a) lumped controls and (b) the case when (1.1) admits multiple solutions. The latter constitutes the classical ill-posedness of the boundary problem (1.1), which, however, is not an unusual situation in the semilinear case. The principal concern here is the following: if the same control can generate several solutions (which one is unknown in advance), then the whole idea of "control" of the physical process it describes does not make much sense. In this respect, we intend to show that, in some cases, one can still "steer" such a system in terms of the following definition, namely, by employing a carefully chosen control, which acts uniformly upon all the solutions, which it possibly generates.

Definition 1.1. Given $T>0$, we shall say that system (1.1), admitting multiple solutions in $C([0, T] ; B)$,

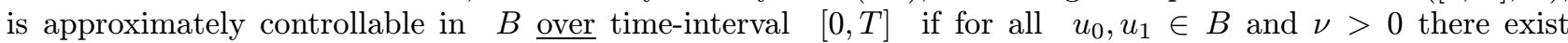
$t_{*}=t_{*}\left(u_{0}, u_{1}, \nu\right) \in[0, T]$ and $v=v\left(u_{0}, u_{1}, \nu\right) \in L^{2}\left(0, t_{*}\right)$ such that

$$
\left\|u_{1}-u\left(\cdot, t_{*}\right)\right\|_{B} \leq \nu
$$

where $u$ is an arbitrary solution to (1.1) corresponding to the selected control $v$ (within the specified class of solutions).

In this definition, the fact that the instant $t_{*}$, when the desirable steering is achieved, is not necessarily equal to $T$ we view as a "payment" for the lack of uniqueness property. This circumstance does not seem essential to us though, because, regardless of $u_{0}$ and $u_{1}$, this $t_{*}$ is bounded by $T$.

The rest of the article is organized as follows. In Section 2 we formulate our main results and the proofs are given in Sections 3-6. 


\section{MAIN RESULTS}

\subsection{The classical case}

We begin with the case when $f=f(u)$.

Theorem 2.1. Let $T>0$ be given and $l_{2} \pm l_{1}$ be the irrational numbers. Suppose that $f=f(u)$ is a continuous function such that

$$
|f(u)| \leq \log ^{\rho}|u| \text { as }|u| \rightarrow \infty,
$$

for some $\rho \in[0,1)$. Then (1.1) is approximately controllable in $L^{2}(0,1)$ over $[0, T]$ in the sense of Definition 1.1.

In the framework of Definition 1.1, here and in Theorems 2.2 and 2.5 below we consider all the possible solutions of (1.1) in the space $C\left([0, T] ; L^{2}(\Omega)\right) \cap H_{0}^{1,0}\left(Q_{T}\right)$ defined via the corresponding integral identity, as described in the existence result of [10], pp. 466-467.

\subsection{Controllability in $L^{2}(0,1)$}

Our next results deal with the case when $f=f(x, t, u)$. More precisely, we are concerned with the question on how the regularity of $f(x, t, u)$ in $x$ and $t$ and its structure affect the growth rate of $f$ in $u$, required to ensure the desirable approximate controllability property. We distinguish three principal components which contribute here: (a) the existence theory, which is closely related to (b) the regularity theory for the semilinear pde's (see, e.g. $[10,14])$, and (c) the asymptotic behavior of the norms of controls solving the corresponding truncated linear controllability problem (1.3) as $t \rightarrow 0$.

First of all, let us note that, at no extra cost, Theorem 2.1 can be extended to the "additive" case when

$$
f(x, t, u)=f_{1}(x, t, u)+f_{2}(x, t, u),
$$

where $\left|f_{2}(x, t, u)\right|$ satisfies $(2.1)$ a.e. in $Q_{T},\left|f_{1}(x, t, u)\right| \leq \psi(x, t)$, and $f_{1}, f_{2}$ and $\psi$ are such that $f$ complies with the existence conditions for $(1.1)$ in $C\left([0, T] ; L^{2}(\Omega)\right) \cap H_{0}^{1,0}\left(Q_{T}\right)$.

We now introduce the following assumption. (Note that it is consistent with the classical constraints on $f$ relevant to (1.1), see, e.g. $[10,14]$.)

Assumption 2.1. Suppose that $f(x, t, u)$ is Lebesgue's measurable in $(x, t, u)$, continuous in $u$ for almost all $(x, t) \in Q_{T}$, and is such that

$$
|f(x, t, u)| \leq \varphi(x, t)+f^{*}(|u|) \text { a.e. in } Q_{T} \quad \forall u \in R,
$$

where $f^{*}$ is a continuous nonnegative monotone nondecreasing function on $R_{+}=\{a \mid a \in R, a \geq 0\}$. (Other conditions on $\varphi$ and $f$ will be given below.)

To satisfy $(2.2), f$ does not need to be of the additive form. Indeed, if $f(x, t, u)=f_{1}(x, t, u) f_{2}(x, t, u)$, then, by Young's inequality, for any $s>0$ and $p>1$, we have:

$$
|f(x, t, u)| \leq \frac{1}{p} s^{p}\left|f_{1}(x, t, u)\right|^{p}+\frac{p-1}{p} s^{-\frac{p}{p-1}}\left|f_{2}(x, t, u)\right|^{\frac{p}{p-1}},
$$

which is consistent with the form (2.2).

We begin by the case $B=L^{2}(0,1)$. We need the following additional conditions on $f$.

Assumption 2.2. Let $T>0$ be given and, in addition to (2.2), $f(x, t, u)$ satisfy

$$
|f(x, t, u)| \leq \varphi(x, t)+d|u| \text { a.e. in } Q_{T} \quad \forall u \in R,
$$


for some $d>0$ and $\varphi \in L^{6 / 5}\left(Q_{T}\right) \bigcap L^{r_{*}, r}\left(Q_{T}\right) \bigcap L^{q_{1}, q_{2}}\left(Q_{T}\right)$, where $q_{1} \in[1, \infty], q_{2} \in[1,2], r_{1} \in[2, \infty], r_{2} \in$ $[4, \infty]$, and $\beta \in[0,1]$ are such that:

$$
\begin{aligned}
& \frac{1}{2 q_{1}}+\frac{1}{q_{2}}<1-\frac{(1-\beta)}{4}, \frac{1}{q_{1}} \leq 1-\frac{1-\beta}{r_{1}}, \quad \frac{1}{q_{2}}<1-\frac{1-\beta}{r_{2}}-\frac{\beta}{4}, \quad \frac{1}{2 r_{1}}+\frac{1}{r_{2}}=\frac{1}{4}, \\
& \frac{1}{2 r_{*}}+\frac{1}{r}=\frac{5}{4}, \quad r_{*} \in[1,2], \quad r \in[1,4 / 3] .
\end{aligned}
$$

In the above $L^{q_{1}, q_{2}}\left(Q_{T}\right)=L^{q_{2}}\left(0, T ; L^{q_{1}}(0,1)\right)$.

Theorem 2.2. Let $T>0$ be given and $l_{2} \pm l_{1}$ be irrational. Suppose that Assumptions 2.1 and 2.2 hold and that in (2.2)

$$
\int_{0}^{\tau}\left(f^{*}\left(s^{-1 / 4} e^{\alpha / \tau}\right)\right)^{r} d s \rightarrow 0 \text { as } \tau \rightarrow 0^{+} \quad \forall \alpha>0,
$$

where $r$ is as in (2.3). Then system (1.1) is approximately controllable in $L^{2}(0,1)$ over $[0, T]$ in the sense of Definition 1.1. If, in addition, the origin is a stable equilibrium for (1.1), then the corresponding steering can always be achieved at time $T$ exactly.

In the above the term "stable equilibrium" means that for every $\varepsilon>0$ there is a $\delta>0$ such that if $\left\|u_{0}\right\|_{L^{2}(0,1)}<\delta$, then $\|u(\cdot, t)\|_{L^{2}(0,1)}<\varepsilon$ for all $t>0$. For example, this is the case when $f(x, t, u) u \geq 0$ for all $u \in R$ and $(x, t) \in Q_{T}$.

In Theorem 2.2 the best possible case occurs when $r=1$. To explain this, let us recall that our method deals with the limit passage (1.5). We evaluate the expression on the left of (1.5) in terms of the "smallest possible" $L^{p_{1}, p_{2}}\left(Q_{t}\right)$-norm of $f\left(\cdot, \cdot, u_{(t)}(\cdot, \cdot)\right)$ (with $p_{1} \geq 1, p_{2} \geq 1$ ) as $t$ tends to zero. This gives us $p_{2}=r=1$ as the best possible choice (see (4.1) and Step 4 in the proof of Th. 2.2 for details).

On the other hand, the parameter $r$ in (2.4) also depends on the regularity of $\varphi$ in $x$, as given by (2.3). That is, if the regularity of $\varphi$ in $x$ is not "good enough", one has to select $r$ larger than 1 (see also (4.1) below).

Condition (2.4) holds if, for example,

$$
f^{*}(u) \leq \log ^{\rho} u \text { as } u \rightarrow \infty,
$$

where $\rho \in[0,1 / r)$. Indeed, in this case for "small" $\tau$ and $0<s<\tau$ we have:

$$
f^{*}\left(s^{-1 / 4} e^{\alpha / \tau}\right) \leq \log ^{\rho}\left(s^{-1 / 4} e^{\alpha / \tau}\right) \leq\left(-\frac{1}{4} \log s\right)^{\rho}+\left(\frac{\alpha}{\tau}\right)^{\rho} \leq \frac{1}{4^{\rho}}\left|\log ^{\rho} s\right|+\frac{\alpha^{\rho}}{\tau^{\rho}} .
$$

Here we used the inequality $(a+b)^{\gamma} \leq C(\gamma)\left(a^{\gamma}+b^{\gamma}\right), \forall a, b, \gamma \geq 0$ for some $C(\gamma)>0 \quad(C(\gamma)=1$ for $0 \leq \gamma \leq 1)$. Then (again as $\tau \rightarrow 0$ ):

$$
\left(f^{*}\left(s^{-1 / 4} e^{\alpha / \tau}\right)\right)^{r} \leq \frac{C(r)}{4^{r \rho}}|\log s|^{r \rho}+\frac{C(r) \alpha^{r \rho}}{\tau^{r \rho}} \leq C(r) \frac{1}{4^{r \rho}}|\log s|+\frac{C(r) \alpha^{r \rho}}{\tau^{r \rho}},
$$

from which (2.4) follows, since $r \rho<1$. 


\section{Remark 2.1.}

- In [9] the approximate controllability of $(1.1)$ was shown in $L^{2}(\Omega)$ over any $[0, T]$ for the reaction-diffusionconvection nonlinearity $f=f\left(x, t, u, u_{x}\right)$, while assuming that $u_{0} \in L^{\infty}(0,1)$ and that $(2.2)$ holds for $\varphi \in L^{6 / 5}\left(Q_{T}\right)$ and $f_{*}(|u|) \leq[\log \log |u|]^{\rho}, \rho \in[0,5 / 6)$ as $|u| \rightarrow \infty$. This result was based on the $L^{\infty}$-estimate technique of [10] for this class of equations (i.e., with convection terms).

- In our framework, condition (2.4) seems to be optimal in the sense that: (a) via (2.3) Assumption 2.2 takes into account the sharp results on $L^{\infty}$-estimates for (1.1) (see [14]); (b) in [16] it was shown that the estimate (4.5) below, which is "responsible" for the appearance of the exponential dependence in (2.4), is sharp; and (c) parameters $6 / 5$ and $r$ arise in the sharp existence and regularity results for solutions to (1.1) in $C\left([0, T] ; L^{2}(\Omega)\right) \bigcap H_{0}^{1,0}\left(Q_{T}\right)[10]$. Accordingly, in the embedding $\left.\varphi \in L^{6 / 5}\left(Q_{T}\right) \bigcap L^{r_{*}, r}\left(Q_{T}\right) \bigcap L^{q_{1}, q_{2}}\left(Q_{T}\right)\right)$ the first space is to guarantee the existence of solutions to (1.1) ( [10], pp. 466-467), while the second and the third ones are to use the sharp energy estimate (4.1) from [10] and suitable $L^{\infty}$-estimates from [14].

\subsection{Controllability in $C_{0}[0,1]$ and $L^{\infty}(0,1)$}

Because of the role they play for the parabolic equations (we refer, e.g., to the maximum principle), the steering of solutions to (1.1) in these spaces is of special interest. However, it is known that with $u_{0} \in L^{\infty}(0,1)$ one can have $u \notin C\left([0, T], L^{\infty}(0,1)\right)$. Indeed, the semigroup $S(t)$ is not strongly continuous on $L^{\infty}(0,1)$ and

$$
\lim _{t \rightarrow 0}\left\|S(t) u_{0}-u_{0}\right\|_{L^{\infty}(0,1)}=0
$$

if and only if $u_{0} \in C_{0}[0,1]$, see, e.g. [14], pp. 19-20. Formally, this does not allow the use of Definition 1.1. On the other hand, $S(t)$ is strongly continuous on $L^{1}(0,1)$, which enables us to define uniquely the initial and target states by using formula (1.2) (i.e., employing the continuity in a less regular $L^{1}(0,1)$ ). Moreover, Lemma 2.1 of [14] (p. 78) implies that under the assumptions of Theorem 2.3 any $L^{\infty}\left(Q_{T}\right)$-solution of the corresponding boundary problem lies in $C\left((0, T] ; C_{0}[0,1]\right)$. Hence, we can use Definition 1.1 with one correction: instead of assuming that $u \in C([0, T] ; B)$, we will further assume that $u \in C\left((0, T] ; L^{\infty}(0,1)\right) \cap C\left([0, T] ; L^{1}(0,1)\right)$ for $B=L^{\infty}(0,1)$.

Assumption 2.3. Let $T>0$ be given and

$$
\frac{1}{2 q_{1}}+\frac{1}{q_{2}}+\mu=1, \quad q_{1}, q_{2} \in[1, \infty], \quad \mu \in(0,1) .
$$

Assume that $f(x, t, u)$ satisfies

$$
|f(x, t, u)| \leq c_{*}(x, t)(1+|u|) \text { a.e. in } Q_{T} \forall u \in R,
$$

for some $c_{*} \in L^{q_{1}, q_{2}}\left(Q_{T}\right)$ and is locally Lipschitz as follows:

$$
\left|f\left(x, t, u_{1}\right)-f\left(x, t, u_{2}\right)\right| \leq c_{*}(x, t) \Gamma\left(\left|u_{1}\right|+\left|u_{2}\right|\right)\left|u_{1}-u_{2}\right| \quad \forall u, v \in R,
$$

where $\Gamma$ is an increasing function.

Let $\mathcal{C}_{0}(0,1)$ be the (closed) subspace of $L^{\infty}(0,1)$, which is generated by all the finite linear combinations of the eigenfunctions associated with the truncated problem (1.3). (Note that $\mathcal{C}_{0}(0,1)$ is, in fact, the space $C_{0}[0,1]=\{w \mid w \in C[0,1], w(0)=w(1)=0\}$, regarded as a subspace of $\left.L^{\infty}(0,1).\right)$ 
Theorem 2.3. Let $T>0$ be given and $l_{2} \pm l_{1}$ be the irrational numbers. Suppose that Assumptions 2.1 and 2.3 hold and in (2.2) $\varphi \in L^{q_{1}, q_{2}}\left(Q_{T}\right)$, and

$$
s^{1-1 / 2 q_{1}} f^{*}\left(e^{\alpha / s}\right) \rightarrow 0 \text { as } s \rightarrow 0^{+} \quad \forall \alpha>0 .
$$

Then for any $u_{0} \in L^{\infty}(0,1), u_{1} \in \mathcal{C}_{0}(0,1)$ and $\nu>0$ there is a control $v \in L^{2}(0, T)$ such that for the corresponding solution $u$ to (1.1) the following estimate holds:

$$
\left\|u_{1}-u(\cdot, T)\right\|_{L^{\infty}(0,1)} \leq \nu
$$

There are no multiple solutions to (1.1) in the case of Theorem 2.3 (see Lem. 5.1 below).

In this result and the following Theorem 2.4 we deal with the solutions to (1.1) described via the semigroup presentation (as in Lem. 5.1 below, see [14], p. 29).

It can be shown that the following condition implies $(2.7)$ :

$$
f^{*}(s) \leq \log ^{\rho} s, \quad \rho \in\left[0,1-1 / 2 q_{1}\right) \text { as } s \rightarrow \infty
$$

The best possible case here $(\rho \in[0,1))$ corresponds to $q_{1}=\infty$. (Note that $q_{1}$ also depends on $q_{2}$ in Ass. 2.3.)

Remark 2.2. One can notice the following difference between (2.8) and (2.5), which we attribute to the change of the space for controllability: (2.5) includes only the parameter $r$ (recall that $r$ depends on $r_{*}$ too) associated with the time-variable, while (2.8) explicitly involves only $q_{1}$, which is associated with the regularity of $f$ in $x$.

Under the assumptions of Theorem 2.3, it is possible to prove that (see [14], p. 41)

$$
\lim _{t \rightarrow 0}\left\|u(\cdot, t)-u_{L}(\cdot, t)\right\|_{L^{\infty}(0,1)}=0 \quad \forall u_{0} \in L^{\infty}(0,1)
$$

Due to $(2.6)$, this yields that $u \in C\left([0, T] ; C_{0}[0,1]\right)$, provided $u_{0} \in C_{0}[0,1]$. Hence, Theorem 2.3 implies the following.

Theorem 2.4. Under the assumptions of Theorem 2.3 system (1.1) is approximately controllable in $C_{0}[0,1]$ at time $T$ in the classical sense.

Remark 2.3. The fact that $u \in C\left((0, T] ; C_{0}[0,1]\right)$ under the assumptions of Th. 2.3 suggests that Theorems 2.3 and 2.4 provide the "largest possible" partial approximate controllability results accordingly in $L^{\infty}(0,1)$ and $C[0,1]$.

\subsection{An example of the superlinear growth}

An example due to Bamberger (see also [4, 7], p. 51) showed that when $f(u)=u|u|^{r}, r>0$ the approximate controllability of (1.1) is not possible in any of $L^{p}(\Omega), 1 \leq p<\infty$ at any positive time, even by the locally distributed controls. More precisely, it turns out that in this case the control impact does not propagate "effectively" from $\omega$ to the rest of the space domain: regardless of how large is the control applied on $\omega$, the corresponding solutions remain uniformly bounded on any closed subset of $\Omega \backslash \bar{\omega}$. From here we have to conclude that if the global approximate controllability of $(1.1)$ is possible in $L^{2}(\Omega)$ for some superlinear terms, then they must satisfy certain assumptions which exclude Bamberger's case. In this respect we give the following example dealing with the restrictions on the growth of nonlinear term in $t$, which allows us, by applying a suitably chosen "quick" control, to neglect the effect of nonlinearity. (Recall that the above mentioned examples do not satisfy such a restriction.) 
Consider the following reaction-diffusion-convection equation:

$$
\begin{aligned}
u_{t} & =u_{x x}-f\left(x, t, u, u_{x}\right)+v(t) \chi_{\left(l_{1}, l_{2}\right)}(x) \text { in } Q_{T}, v \in L^{2}(0, T), \\
u(0, t) & =u(1, t)=0, \quad u(x, 0)=u_{0}(x) .
\end{aligned}
$$

We assume that $f(x, t, u, p)$ is Lebesgue's measurable in $(x, t, u, p)$ and continuous in $(u, p)$ for almost all $(x, t) \in Q_{T}$, and is such that

$$
\begin{gathered}
|f(x, t, u, p)| \leq \varphi(x, t)+\beta(t)|u|^{d_{1}}+\beta(t)|p|^{d_{2}} \quad \text { a.e. in } Q_{T} \text { for } u, p \in R, \\
\int_{0}^{1} f\left(x, t, \phi, \phi_{x}\right) \phi d x \geq(\nu-1) \int_{0}^{1} \phi_{x}^{2} d x-\rho(t) \int_{0}^{1}\left(1+\phi^{2}\right) d x \quad \forall \phi \in H_{0}^{1}(0,1),
\end{gathered}
$$

where $\beta(t)$ is a non-negative monotone nondecreasing continuous function, $d_{1} \in[0,5), d_{2} \in[0,5 / 3), \quad \nu>$ $0, \rho(\cdot) \in L^{1}(0, T), \int_{0}^{T} \rho(t) d t \leq \beta(T)$, and $\varphi \in L^{6 / 5}\left(Q_{T}\right)$. These conditions ensure the existence of at least one generalized solution of $(2.9)$ in $C\left([0, T] ; L^{2}(\Omega)\right) \cap H_{0}^{1,0}\left(Q_{T}\right)$ (e.g. [10], pp. 466-467). However its uniqueness is not guaranteed. Our method provides the following result for this equation.

Theorem 2.5. Given $T>0$, assume that $l_{2} \pm l_{1}$ are irrational, and that

$$
e^{\xi / t} \beta(t) \rightarrow 0 \text { as } t \rightarrow 0 \quad \forall \xi>0
$$

Then system (2.9) is approximately controllable in $L^{2}(0,1)$ over $[0, T]$ in the sense of Definition 1.1.

\section{PRELIMinaRies}

\subsection{The linear case revisited}

Here we give a very brief account of the approximate controllability results available for the linear truncated problem (1.3). Our goal is to explain the specific attention to the one dimensional case.

Let $\lambda_{k}=(\pi k)^{2}$ and $\omega_{k}(x)=\sqrt{2} \sin \pi k x, k=1, \ldots$ denote the eigenvalues and eigenfunctions (orthonormalized in $\left.L^{2}(0,1)\right)$ for (1.3). It was shown in [15] that (1.3) is approximately controllable in $L^{2}(0,1)$ at (any) time $T>0$ if and only if the averaged values $\int_{\omega} \omega_{k}(x) d x \neq 0$ for $k=1, \ldots$ Since (1.3) is a particular case of (1.1), this explains why we assume in Theorems 2.1-2.5 that $l_{2} \pm l_{1}$ are the irrational numbers.

As a matter of fact, if the multiplicities of the corresponding eigenvalues are uniformly bounded, then the result of [15] can be extended to the general $n$-dimensional case. This restrictive assumption can, however, be avoided by introducing the mobile point controls [8].

The argument in [15] is based on the duality and analytic continuation techniques. The latter is employed to prove the corresponding unique continuation result from the averaged over $\left(l_{1}, l_{2}\right)$ traces of solutions to the linear system dual of (1.3). Generally, this approach does not give a constructive description of the controls involved, though they can always be described through the solutions of suitable optimal control problems [11].

In the one space dimension a comprehensive constructive linear controllability theory has been developed in $[1,5,12,13]$, based on the idea of Riesz basis. However, it does not apply in higher dimensions. To explain this, let us recall that only for $n=1$ one has $\sum_{k=1}^{\infty} \frac{1}{\lambda_{k}}<\infty$ and $\left|\lambda_{k}-\lambda_{m}\right| \geq \rho|k-m|$ with $\rho>0$, in which case $\left\{e^{-\lambda_{k} t} \mid k=1, \ldots\right\}$ form a Riesz basis in their span in $L^{2}(0, T)$ for any $T>0[5,12,13,18]$. This in turn makes it possible to solve the linear approximate controllability problem explicitly in terms of controls that are the finite linear combinations of the elements of the corresponding biorthogonal sequence. This explicit solution is crucial in our analysis, which centers around the study of its asymptotic behavior as $T \rightarrow 0$ (see, e.g. (4.5) to this end). In contrast to that, the Riesz basis property is out of question for $n>1$, because in this case 
for every finite set $J$ of natural numbers and every $T>0$ the functions $\left\{e^{-\lambda_{k} t} \mid k=1, \ldots, k \notin J\right\}$ span all the spaces $C[0, T], L^{p}(0, T), p \in[1, \infty)$, see, e.g. [12], p. 30 .

We will need the following formulae describing the solution to the linear approximate controllability problem (1.3). They can easily be derived, e.g., based on the ideas of $[5,13]$.

It is well known that the solution of (1.3) admits the following representation:

$$
\begin{aligned}
u_{L}(x, t)= & \sum_{k=1}^{\infty} e^{-\lambda_{k} t}\left(\int_{0}^{1} u_{0}(r) \omega_{k}(r) d r\right) \omega_{k}(x) \\
& +\sum_{k=1}^{\infty}\left(\int_{0}^{t} e^{-\lambda_{k}(t-\tau)} v(\tau)\left(\int_{0}^{1} \chi_{\left[l_{1}, l_{2}\right]}(r) \omega_{k}(r) d r\right) d \tau\right) \omega_{k}(x) .
\end{aligned}
$$

Let $\left\{q_{k}(\cdot, t)\right\}_{k=1}^{\infty}$ be a biorthogonal sequence to $\left\{e^{\lambda_{k} \tau}\right\}_{k=1}^{\infty}$ in $L^{2}(0, t)[5,12,18]$ :

$$
\int_{0}^{t} e^{-\lambda_{k} \tau} q_{l}(\tau, t) d \tau= \begin{cases}1, & \text { if } k=l \\ 0, & \text { if } k \neq l .\end{cases}
$$

Then, provided the numbers $l_{2} \pm l_{1}$ are irrational, the controls

$$
v_{(t), k}(\tau)=q_{k}(t-\tau, t)\left(\sqrt{2} \int_{l_{1}}^{l_{2}} \sin \pi k x d x\right)^{-1}, \tau \in(0, t)
$$

solve the following moment problem:

$$
\int_{0}^{t} e^{-\lambda_{l}(t-\tau)}\left(\int_{l_{1}}^{l_{2}} v_{(t), k}(\tau) \omega_{l}(x) d x\right) d \tau= \begin{cases}1, & \text { if } l=k \\ 0, & \text { if } l \neq k\end{cases}
$$

For every $t \in(0, T]$ denote by $V_{*}(0, t)$ the set of all finite linear combinations of $v_{(t), k}, k=1, \ldots$ Since $\left\{\omega_{k}\right\}_{k=1}^{\infty}$ form a basis in $L^{2}(0,1)$, formulae (3.1-3.3) allow one to conclude that (1.3) is approximately controllable in the classical sense in $L^{2}(0,1)$ at any time $t \in(0, T]$ by using controls $v \in V_{*}(0, t)$ only.

\subsection{The general scheme of further proofs}

Our general strategy (as well as the one in of the above-referred works) is to view the semilinear boundary problem as a "disturbed version" of the linear one. In this way, assuming that the latter already possesses a desirable controllability property, sufficient conditions for controllability of the former are sought as suitable structural and regularity assumptions on nonlinear terms. In that respect, the more information is available on the linear controllability problem the "better" results can be expected in the semilinear case. Therefore, as Section 3.1 implies, the main attention is given below to the one dimensional semilinear reaction-diffusion equation.

The idea of our method, as it is fully described in Section 1.2, centers around the proof of the limit passage (1.5) for suitably chosen dense set of targets $u_{1}$ 's. More precisely, in the case of Theorems 2.1 and 2.2 it can be described as follows. 
Take any instant $t \in(0, T]$ and consider arbitrary $u_{0} \in L^{2}(0,1)$ and $u_{1}=u_{0}+\sum_{k=1}^{K} \alpha_{k} \omega_{k}$. Put $v_{(t)}=\sum_{k=1}^{K} \alpha_{k} v_{(t), k} \in V_{*}(0, t)$. Then we have:

$$
u_{(t)}(\cdot, t)-u_{L,(t)}(\cdot, t)=-\int_{0}^{t} S(t-\tau) f\left(\cdot, \tau, u_{(t)}\right) d \tau, \quad u_{L,(t)}(\cdot, t)=S(t) u_{0}+\sum_{k=1}^{K} \alpha_{k} \omega_{k}, \quad \forall t \in(0, T],
$$

where $u_{(t)}$ and $u_{L,(t)}$ are accordingly a solution to (1.1) (i.e., any of possible associated multiple solutions) and the unique solution to $(1.3)$ on $(0, t)$, corresponding to $u_{0}$ and $v_{(t)}$.

Taking into account that (1.3) is approximately controllable, we can view the right-hand side of the first equality above as an "error" of steering of (1.1). Then, if we manage to show that, uniformly over all the aforementioned possible solutions $u_{(t)}$,

$$
\lim _{t \rightarrow 0}\left\|u_{(t)}-u_{L,(t)}\right\|_{C\left([0, t] ; L^{2}(0,1)\right)}=\lim _{t \rightarrow 0} \max _{r \in[0, t]}\left\|\int_{0}^{r} S(r-\tau) f\left(\cdot, \tau, u_{(t)}\right) d \tau\right\|_{L^{2}(0,1)}=0,
$$

then the states $u_{(t)}(\cdot, t)$ uniformly approach $u_{1}=u_{0}+\sum_{k=1}^{K} \alpha_{k} \omega_{k}=\lim _{t \rightarrow 0} u_{L,(t)}(\cdot, t)$ as $t$ tends to zero. On the other hand, since for any $u_{0} \in L^{2}(\Omega)$ the set of such functions $u_{1}$ is dense in $L^{2}(\Omega),(3.4)$ implies (1.5), that is, the first part of Theorem 2.2.

Actually, it is sufficient only to show that, for any of $k=1, \ldots$, one has

$$
\lim _{t \rightarrow 0}\left\|u_{(t), k}-u_{L(t), k}\right\|_{C\left([0, t] ; L^{2}(0,1)\right)}=\lim _{t \rightarrow 0} \max _{r \in[0, t]}\left\|\int_{0}^{r} S(r-\tau) f\left(\cdot, \tau, u_{(t), k}\right) d \tau\right\|_{L^{2}(0,1)}=0
$$

where $u_{(t), k}$ and $u_{L(t), k}$ are accordingly any associated solution to (1.1) and the unique solution to (1.3) on $(0, t)$ with $v=v_{(t), k}$. This replacement of (3.4) with (3.5) is possible, because the proof of (3.5) relies below on the explicit estimates in terms of the corresponding norms of controls and $u_{0}$. Therefore, thanks to the triangle inequality, the estimates for any $v_{(t)}$ in $V_{*}(0, t)$ are immediate.

\section{Proofs of Theorems 2.1 and 2.2}

Proof of Theorem 2.1. This is a particular case of Theorem 2.2, in which we need to select the function $\varphi$ as the constant function and put $r=1$. One needs to use first the classical regularity estimate for solutions to (1.1):

$$
\left\|\int_{0}^{r} S(r-\tau) f\left(\cdot, \tau, u_{(t), k}\right) d \tau\right\|_{L^{2}(0,1)} \leq c\left\|f\left(u_{(t), k}\right)\right\|_{L^{2,1}\left(Q_{t}\right)} \quad \forall t \in[0, T]
$$

where $c$ is a positive constant. Then, the estimate (4.6) provides (2.1). Hence, the crux of the proof is the derivation of (4.6), which combines Lemma 4.2 from [9] with suitable regularity results from [10] and $L^{\infty}$-estimates from [14]. To avoid repeating, we derive this estimate in the general case in the proof of Theorem 2.2. Indeed, the only difference here is that a particular choice of the function $\varphi$ defines the choice of parameters in Assumption 2.2, required to apply the further, otherwise identical, estimates.

Proof of Theorem 2.2. Note first of all that the existence of a solution to $(1.1)$ in $C\left([0, T] ; L^{2}(\Omega)\right) \cap H_{0}^{1,0}\left(Q_{T}\right)$ follows from [10], pp. 466-467. We now proceed with the derivation of (3.5).

Step 1. Note that we have:

$$
\left(u-u_{L}\right)_{t}=\left(u-u_{L}\right)_{x x}-f(x, t, u) \text { in } Q_{T},\left.\quad\left(u-u_{L}\right)\right|_{x=0,1}=0,\left.\quad\left(u-u_{L}\right)\right|_{t=0}=0 .
$$


Take any $v=v_{(t), k}$. The following energy estimate is classical $([10]$, p. 139, 135) for this linear problem:

$$
\left\|u_{(t), k}-u_{L(t), k}\right\|_{C\left([0, t] ; L^{2}(0,1)\right)} \leq c^{*}(t)\left\|f\left(\cdot, \cdot, u_{(t), k}\right)\right\|_{L^{r_{*}, r}\left(Q_{t}\right)} \quad \forall t \in[0, T] .
$$

Here and below, we use $c^{*}=c^{*}(\cdot)$ to denote a generic positive nondecreasing function on $R_{+}$.

We will also need the following energy estimate from [9].

Lemma 4.1 ([9], p. 863). Under the assumptions of Theorem 2.2 any solution of (1.1) in $C\left([0, T] ; L^{2}(\Omega)\right)$ $\bigcap H_{0}^{1,0}\left(Q_{T}\right)$ satisfies the following two estimates

$$
\begin{aligned}
& \|u\|_{L^{6}\left(Q_{t}\right)} \leq c^{*}(t)\left(\left\|u_{0}\right\|_{L^{2}(0,1)}+\sqrt{t}\|v\|_{L^{2}(0, t)}+1\right), \quad \forall t \in[0, T] \\
& |u|_{Q_{t}}=\|u\|_{C\left([0, t] ; L^{2}(\Omega)\right)}+\left(\int_{0}^{t} \int_{\Omega} u_{x}^{2} d x d t\right)^{1 / 2} \leq c^{*}(t)\left(\left\|u_{0}\right\|_{L^{2}(0,1)}+\sqrt{t}\|v\|_{L^{2}(0, t)}+1\right), \quad \forall t \in[0, T] .
\end{aligned}
$$

Step 2. To establish (3.5), we need to evaluate the right-hand side of (4.1). To this end, we will use some results on a priori $L^{\infty}$-estimates in [14].

Recall that $f(\cdot, \cdot, u) \in L^{q^{\prime}}\left(Q_{T}\right)$ (see [10], p. 469). Hence, any solution of $(1.1)$ in the space $C\left([0, T] ; L^{2}(\Omega)\right.$ ) $\bigcap H_{0}^{1,0}\left(Q_{T}\right)$ is also a mild solution in the sense of [14], p. 29, namely:

$$
\begin{gathered}
u(\cdot, t) \in L^{1}(0,1) \text { for } t \in(0, T) ; \quad-f(\cdot, \cdot, u)+v(\cdot) \chi_{\left[l_{1}, l_{2}\right]}(\cdot) \in L^{1}\left(Q_{T}\right) ; \\
u(\cdot, t)=S(t) u_{0}+\int_{0}^{t} S(t-\tau)\left(f(\cdot, \tau, u)+v(\tau) \chi_{\left[l_{1}, l_{2}\right]}\right) d \tau \text { for } t \in(0, T) .
\end{gathered}
$$

This permits us to use suitable results from [14] dealing with mild solutions.

We intend to apply Lemma 19 of [14], p. 69 (note that in [14] the notation $n=1 / 2$ is used). There we are concerned with the following system of parameters:

$$
\begin{aligned}
& \frac{1}{2 q_{1}}+\frac{1}{q_{2}}<1+(1-\gamma \beta) \frac{1}{2 p}-\gamma(1-\beta)\left(\frac{1}{2 r_{1}}-\frac{1}{r_{2}}\right)-\varepsilon, \\
& \frac{1}{q_{1}} \leq 1-\frac{\gamma(1-\beta)}{r_{1}}-\frac{\gamma \beta}{p}, \quad \frac{1}{q_{2}}<1-\frac{\gamma(1-\beta)}{r_{2}}-\gamma \beta \delta-\varepsilon, \quad \delta=\frac{1}{2 p_{0}}-\frac{1}{2 p}, \quad \beta \gamma<1,
\end{aligned}
$$

where $q_{1}, q_{2}, r_{1}$, and $r_{2}$ are as in Theorem 2.1. To satisfy the other restrictions in [14] and Assumption 2.2, we select $\gamma=1$ (compare with the hypothesis (F1) in [14], p. 13), $p_{0}=2, p=\infty$, and take any $\varepsilon \in(0,1)$.

Recall now that $C\left([0, T] ; L^{2}(\Omega)\right) \cap H_{0}^{1,0}\left(Q_{T}\right)$ is continuously embedded into $L^{r_{1}, r_{2}}\left(Q_{T}\right)$ (see, e.g. [10], p. 466). Combining this with (4.2), we deduce,

$$
\|u\|_{L^{r_{1}, r_{2}}\left(Q_{t}\right)} \leq C(t)\left(\left\|u_{0}\right\|_{L^{2}(0,1)}+\sqrt{t}\|v\|_{L^{2}(0, t)}+1\right)<\infty, \quad \forall t \in[0, T],
$$

which gives us the condition (212) on p. 69 of [14], required to make use of Lemma 19 in [14].

To be consistent with this lemma, we obtain from the first first condition in Assumption 2.2:

$$
\left|f(x, t, u)+v(t) \chi_{\left[l_{1}, L_{2}\right]}(x)\right| \leq(\varphi(x, t)+d+|v(t)|)(1+|u|) .
$$

Here and below, for simplicity, we regard $v=v(t)$ both as a function on $(0, T)$ and on $Q_{T}$. Then, $\varphi+d+|v(t)| \in$ $L^{q_{1}, q_{2}}\left(Q_{T}\right)$. Furthermore, from formula (214) on p. 69 of [14] (with $\delta=1 / 4$, see above), it follows that for 
almost all $s \in[0, t]$ the following inequality holds:

$$
\begin{aligned}
\|u(\cdot, s)\|_{L^{\infty}(0,1)} & \leq K m^{-1 / 4}(s)\left\{\left\|u_{0}\right\|_{L^{2}(0,1)}+m^{\varepsilon}(t)\left[\|\varphi+d+|v|\|_{L^{q_{1}, q_{2}}\left(Q_{t}\right)}\left(1+\|u\|_{L^{r_{1}, r_{2}\left(Q_{t}\right)}}\right)^{(1-\beta)}\right.\right. \\
& \left.+\|\varphi+d+|v|\|_{L^{q_{1}, q_{2}\left(Q_{t}\right)}}^{1 /(1-\beta)}\left(1+\|u\|_{L^{r_{1}, r_{2}}\left(Q_{t}\right)}\right]\right\}
\end{aligned}
$$

where $m(t)=\min \{1, t\}$ and $K$ does not depend on $v, t$, and $\varepsilon$.

Step 3. We now need the following lemma, which is proven in [9], p. 874.

Lemma 4.2 [9]. There exists a sequence of positive constants $\left\{\beta_{k}\right\}_{k=1}^{\infty}$ such that:

$$
\left\|v_{(t), k}\right\|_{L^{2}(0, t)} \leq e^{\beta_{k} / t} \quad \text { as } \quad t \rightarrow 0, \quad k=1, \ldots
$$

Remark 4.1. In a different form the estimates analogous to (4.5) were obtained in [16], where it was also shown that this type of exponential time-dependence is sharp.

By Hölder's inequality,

$$
\left(\int_{0}^{t} v^{q_{2}}(\tau) d \tau\right)^{1 / q_{2}} \leq\left(\int_{0}^{t} v^{2}(\tau) d \tau\right)^{1 / 2}\left(\int_{0}^{t} d \tau\right)^{\left(1-q_{2} / 2\right) / q_{2}} \leq t^{\left(1-q_{2} / 2\right) / q_{2}}\|v\|_{L^{2}(0, t)} .
$$

For $v=v_{(t), k}$, this estimate, being combined with (4.5), gives:

$$
\left\|v_{(t), k}\right\|_{L^{q_{1}, q_{2}}(0, t)} \leq e^{L_{k} / t} \quad \text { as } \quad t \rightarrow 0, \quad k=1, \ldots
$$

for some positive $L_{k}$ 's. In turn, applying (4.5) to (4.3) yields:

$$
\left\|u_{(t), k}\right\|_{L^{r_{1}, r_{2}}\left(Q_{t}\right)} \leq e^{M_{k} / t} \quad \text { as } \quad t \rightarrow 0, \quad k=1, \ldots
$$

for some positive $M_{k}$ 's.

Applying these two new estimates to the right-hand side of (4.4) yields that for almost all $s \in[0, t]$ :

$$
\left\|u_{(t), k}(\cdot, s)\right\|_{L^{\infty}(0,1)} \leq K s^{-1 / 4} e^{\alpha_{k} / t} \quad \text { as } t \rightarrow 0 \quad k=1, \ldots
$$

for some positive $\alpha_{k}$ 's.

Step 4. By (2.2),

$$
\left.\left\|f\left(\cdot, \cdot, u_{(t), k}\right)\right\|_{L^{r_{*}, r}\left(Q_{t}\right)} \leq\left\|\varphi^{*}\right\|_{L^{r_{*}, r}\left(Q_{t}\right)}+\left(l_{2}-l_{1}\right)^{1 / r_{*}}\left(\int_{0}^{t} f^{*}\left(\left\|u_{(t), k}(\cdot, s)\right\|_{L^{\infty}(0,1)}\right)\right)^{r} d s\right)^{1 / r} .
$$

Combining (4.7) and (4.6) yields that, for some $\alpha_{1 k}, \alpha_{2 k}>0$,

$$
\left\|f\left(\cdot, \cdot, u_{(t), k}\right)\right\|_{L^{r_{*}, r}\left(Q_{t}\right)} \leq\left\|\varphi^{*}\right\|_{L^{r_{*}, r}\left(Q_{t}\right)}+\left(l_{2}-l_{1}\right)^{1 / r_{*}}\left(\int_{0}^{t}\left(f^{*}\left(\alpha_{1 k} s^{-1 / 4} e^{\alpha_{2 k} / t}\right)\right)^{r} d s\right)^{1 / r}
$$

as $t \rightarrow 0$. In view of Assumption 2.2 and (4.1), this implies (3.5). 
Step 5. Observe now that all the estimates in the above are given explicitly in terms of $u_{0}$ and $v / v_{(t), k}$. Hence, it is not difficult to derive that they must hold for any realization of a (possibly non-unique) solution to (1.1). This yields the required approximate controllability in the sense of Definition 1.1.

Step 6. The following observation is used to prove the second part of Theorem 2.2.

Remark 4.2. If in (1.1) and (3.1) the initial conditions are different, say, $u_{01}$ and $u_{02}$, then for any $v=v_{(t), k}$ we have

$$
u_{(t), k}(\cdot, t)-u_{L(t), k}(\cdot, t)=S(t)\left(u_{01}-u_{02}\right)-\int_{0}^{t} S(t-\tau) f\left(\cdot, \tau, u_{(t), k}\right) d \tau .
$$

In contrast to above, now $u_{(t), k}$ and $u_{L(t), k}$ are accordingly any associated solution to (1.1) and the unique solution to $(1.3)$ on $(0, t)$, corresponding respectively to $v=v_{(t), k}$ and $u_{0}=u_{01}$ and to $v=v_{(t), k}$ and $u_{0}=u_{02}$. In the above proof, we established that (3.5) holds for any $u_{0}$. Hence, the same convergence property holds for the second term on the right in (4.8):

$$
\lim _{t \rightarrow 0} \max _{r \in[0, t]}\left\|\int_{0}^{r} S(r-\tau) f\left(\cdot, \tau, u_{(t), k}\right) d \tau\right\|_{L^{2}(0,1)}=0 .
$$

Therefore (1.1) can be steered over an arbitrary small time-interval (in the sense of Def. 1.1) into any prescribed neighborhood of $u_{1}$ from any $u_{01}$ lying in a sufficiently small neighborhood of $u_{02}$ (this makes the first term on the right in (4.8) "small"). Indeed, one just needs to apply the same control which drives (3.1) from $u_{02}$ sufficiently close to $u_{1}$.

Sketch of the proof of the second part of Theorem 2.2. Let $u_{0}, u_{1} \in L^{2}(\Omega)$ be given. If (1.1) has a stable equilibrium at the origin, then the steering at $t=T$ can be achieved as follows. Firstly (as described in Steps 1-5) we steer (1.1) at some time $t_{*}<T$ from $u_{0}$ into a "sufficiently small" neighborhood of the origin. Then we use the zero-control on some interval $\left(t_{*}, t^{*}\right), t^{*} \in\left(t_{*}, T\right]\left(t^{*}\right.$ will be selected a little bit later). By stability, all possible (multiple) solutions to (1.1) will stay in this neighborhood until $t=t^{*}$. Therefore, thanks to Remark 4.2, there is a control, which, regardless of the actual realization of a solution, can steer (1.1) (again as in Steps 1-5) over an arbitrary small time-interval $\left[t^{*}, t^{* *}\right]\left(t^{* *} \in\left(t^{*}, T\right]\right)$ into a "sufficiently small" neighborhood of $u_{1}$. If $t^{*}$ is close enough to $T$, applying the zero-control on $\left[t^{* *}, T\right]$, we will have all (i.e., possibly multiple) $u(\cdot, T)$ as close to $u_{1}$ as we wish. (This follows from the continuity solutions to (1.1) in time: one can use an estimate like (6.1) below to show this.) This completes the proof of Theorem 2.2.

Remark 4.3. In Assumption 2.1 the bound 2 in the restriction on $q_{2}$ is due to our choice of the space $L^{2}(0, T)$ for controls. This restriction ensures, e.g., that $v \chi_{\left[l_{1}, l_{2}\right]} \in L^{q_{1}, q_{2}}\left(Q_{T}\right)$ in the estimates (4.4).

\section{Proof of Theorem 2.3}

Lemma 5.1. Let

$$
\frac{1}{2 q_{1}}+\frac{1}{q_{2}}<1, \quad q_{1}, q_{2} \in[1, \infty]
$$

Assume that $f(\cdot, \cdot, u)$ satisfies Assumptions 2.1 and 2.3. Then, for any $T>0, u_{0} \in L^{\infty}(0,1)$, and $v \in L^{2}(0, T)$ there exists a unique solution to (1.1) in $L^{\infty}\left(Q_{T}\right)$.

Proof. It can be achieved by combining some results from [14]. Put $z=u-u_{L}$. Then we obtain:

$$
z_{t}=z_{x x}-\hat{f}(x, t, z) \text { in } Q_{T}=(0,1) \times(0, T), \quad v \in L^{2}(0, T), \quad z(0, t)=z(1, t)=0, \quad z(x, 0)=0,
$$


where $\hat{f}(x, t, z)=f\left(x, t, z+u_{L}\right)$. Thus, instead of the second inequality in Assumption 2.3, we have

$$
|\hat{f}(x, t, z)| \leq\left(c_{*}(x, t)+c_{*}(x, t)\left\|u_{L}\right\|_{L^{\infty}\left(Q_{T}\right)}\right)(1+|u|),
$$

while the local Lipschitz condition holds with the coefficient $\Gamma\left(\left|z_{1}\right|+\left|z_{2}\right|+2\left\|u_{L}\right\|_{L^{\infty}\left(Q_{T}\right)}\right)$. By Lemma 9 of [14] (p. 41), one has the existence and uniqueness of a mild solution to the above boundary problem in $z$ and, hence, to (1.1) on some $Q_{T^{*}}, T^{*} \leq T$. This solution lies in $L^{\infty}\left(Q_{T^{*}}\right)$. To complete the proof, we apply Theorem 5 of [14], p. 76 (see (249) there), which, by an explosion argument, yields that $T^{*}=T$.

Proof of Theorem 2.3. We argue as in Section 3.2, or (1.2), this time under Assumptions 2.1 and 2.3 and with the space $L^{\infty}(0,1)$ in place of $L^{2}(0,1)$. Since $u, u_{L} \in C\left((0, T] ; C_{0}[0,1]\right)$, (instead of $(3.5)$ ) it is sufficient to show that:

$$
\lim _{t \rightarrow 0}\left\|u_{(t), k}-u_{L(t), k}\right\|_{L^{\infty}\left(Q_{t}\right)}=\lim _{t \rightarrow 0} \operatorname{ess}_{\sup _{r \in[0, t]} \|}\left\|\int_{0}^{r} S(r-\tau) f\left(\cdot, \tau, u_{(t), k}\right) d \tau\right\|_{L^{\infty}(0,1)}=0, \quad k=1, \ldots
$$

Our plan is to prove (5.1) along the sequence of estimates (5.6-5.9), the derivation of which makes use of suitable a priori $L^{\infty}$-estimates in [14] and the asymptotic behavior (4.5).

Step 1. From semigroup theory we have the following estimate, see, e.g. (30) in [14], p. 25:

$$
\|S(t) w\|_{L^{\infty}(0,1)} \leq M m^{-1 / 2 q_{1}}(t) e^{-\lambda_{1} t}\|w\|_{L^{q_{1}}(0,1)} \quad \forall w \in L^{q_{1}}(0,1), \quad \forall t>0 .
$$

Here and below, $M$ is a positive generic constant and $\lambda_{1}$ is the principal eigenvalue of the truncated linear problem (1.3).

In view of Assumption 2.3, $f(\cdot, \cdot, u) \in L^{q_{1}, q_{2}}\left(Q_{T}\right)$ and, hence, for almost all $t \in[0, T]$ :

$$
\|S(t-s) f(\cdot, s, u(\cdot, s))\|_{L^{\infty}(0,1)} \leq M m(t-s)^{-1 / 2 q_{1}} e^{-\lambda_{1}(t-s)}\|c(\cdot, s)\|_{L^{q_{1}}(0,1)}\left(1+\|u\|_{L^{\infty}\left(Q_{t}\right)}\right) .
$$

This yields:

$$
\begin{gathered}
\left\|u(\cdot, t)-u_{L}(\cdot, t)\right\|_{L^{\infty}(0,1)} \leq \int_{0}^{t}\|S(t-\tau) f(\cdot, \tau, u)\|_{L^{\infty}(0,1)} d \tau \\
\leq M\left(1+\|u\|_{L^{\infty}\left(Q_{t}\right)}\right) \int_{0}^{t}(t-s)^{-1 / 2 q_{1}} e^{-\lambda_{1}(t-s)}\|c(\cdot, s)\|_{L^{q_{1}(0,1)}} d s \quad \forall t \leq 1 .
\end{gathered}
$$

From Hölder's inequality, namely,

$$
\left\|w_{1} w_{2}\right\|_{L^{r}(0, t)} \leq\left\|w_{1}\right\|_{L^{r_{1}(0, t)}}\left\|w_{2}\right\|_{L^{r_{2}(0, t)}}, \quad \frac{1}{r_{1}}+\frac{1}{r_{2}}=\frac{1}{r}, \quad w_{i} \in L^{r_{i}}(0, t),
$$

where we put $r=1, r_{1}=\left(\mu+1 / 2 q_{1}\right)^{-1}$, and $r_{2}=q_{2}$, it follows that

$$
\left\|u(\cdot, t)-u_{L}(\cdot, t)\right\|_{L^{\infty}(0,1)} \leq M\left(1+\|u\|_{L^{\infty}\left(Q_{t}\right)}\right) t^{\mu}\|c\|_{L^{q_{1}, q_{2}}\left(Q_{t}\right)} \quad \forall t \in[0, T]
$$

(compare with (92) on p. 42 of [14]).

Step 2. We will evaluate now the $L^{\infty}\left(Q_{t}\right)$-norm of $u$ as $t \rightarrow 0$. From classical results for the linear heat equation (see, e.g. [10]), we have:

$$
\left\|u_{L}\right\|_{L^{\infty}\left(Q_{t}\right)} \leq m_{*}\left(\left\|u_{0}\right\|_{L^{\infty}(0,1)}+\|v\|_{L^{2}\left(Q_{t}\right)}\right),
$$


where $m_{*}$ is a positive constant. Combining this and (5.5) yields

$$
\|u\|_{L^{\infty}\left(Q_{t}\right)} \leq M\left(1+\|u\|_{L^{\infty}\left(Q_{t}\right)}\right) t^{\mu}\|c\|_{L^{q_{1}, q_{2}}\left(Q_{t}\right)}+m_{*}\left(\left\|u_{0}\right\|_{L^{\infty}(0,1)}+\|v\|_{L^{2}\left(Q_{t}\right)}\right) .
$$

If $t^{\mu} M\|c\|_{L^{q_{1}, q_{2}}\left(Q_{t}\right)}<1 / 2$, then it follows from (5.6) with $v=v_{(t), k}$ that:

$$
\left\|u_{(t), k}\right\|_{L^{\infty}\left(Q_{t}\right)} \leq 2\left[m_{*}\left(\left\|u_{0}\right\|_{L^{\infty}(0,1)}+\left\|v_{(t), k}\right\|_{L^{2}\left(Q_{t}\right)}\right)+M t^{\mu}\|c\|_{L^{q_{1}, q_{2}}\left(Q_{t}\right)}\right] .
$$

Step 3. Analogously to (5.3), but using (2.2) instead of the second inequality in Assumption 2.3, we deduce that

$$
\begin{gathered}
\|S(t-s) f(\cdot, s, u(\cdot, s))\|_{L^{\infty}(0,1)} \\
\leq M m(t-s)^{-1 / 2 q_{1}} e^{-\lambda_{1}(t-s)}\left(\|\varphi(\cdot, s)\|_{L^{q_{1}}(0,1)}+\left\|f^{*}(|u(\cdot, s)|)\right\|_{\left.L^{q_{1}(0,1)}\right) .} .\right.
\end{gathered}
$$

From this and (5.1), it follows by Hölder's inequality (as in the lines (5.3-5.5)) that (again, with $v=v_{(t), k}$ ) we have:

$$
\lim _{t \rightarrow 0}\left\|u_{(t), k}-u_{L(t), k}\right\|_{L^{\infty}\left(Q_{t}\right)} \leq M t^{\mu}\|\varphi\|_{L^{q_{1}, q_{2}}\left(Q_{t}\right)}+M\left(1-\frac{1}{2 q_{1}}\right)^{-1} t^{1-\frac{1}{2 q_{1}}} f^{*}\left(\left\|u_{(t), k}\right\|_{L^{\infty}\left(Q_{t}\right)}\right)
$$

(we remind that $M$ is generic).

The first term on the right in (5.8) tends to zero as $t \rightarrow 0$. Hence, to establish (5.1) it remains only to show that

$$
t^{1-\frac{1}{2 q_{1}}} f^{*}\left(\left\|u_{(t), k}\right\|_{L^{\infty}\left(Q_{t}\right)}\right) \rightarrow 0 \text { as } t \rightarrow 0 .
$$

This follows from (5.7), combined with (4.5) and (2.7). This completes the proof of the approximate controllability of (1.1) in the sense of Definition 1.1, that is, over any $[0, T]$. From here, the statement in Theorem 2.3 follows by the argument of Section 1.2.

\section{Remark 5.1.}

- In Step 2 the inequality $t^{\mu} M\|c\|_{L^{q_{1}, q_{2}}\left(Q_{t}\right)}<1 / 2$ can be obtained for $\mu=0$ as $t \rightarrow 0$. However, applying Hölder's inequality in the above requires $\mu \in(0,1)$ (otherwise, one can have the diverging integral).

- In the proof of Theorem 2.3, the local Lipschitz condition has been used merely to prove the existence of a unique solution in $L^{\infty}\left(Q_{T}\right)$.

Proof of Theorem 2.5. Following the discussion in Section 3, we need to show (3.5).

We first recall the estimate (2.10) from [9], p. 865 (see also (6.50) from [10], p. 469):

$$
\left\|u_{(t), k}-u_{L(t), k}\right\|_{C\left([0, t] ; L^{2}(0,1)\right)} \leq c^{*}(t)\|\varphi\|_{L^{6 / 5}\left(Q_{t}\right)}+\beta(t) c^{*}(t)\left(t^{\frac{5}{6}\left(1-\frac{d_{1}}{5}\right)}\|u\|_{L^{6}\left(Q_{t}\right)}^{5 q^{*} / 6}+t^{\frac{5}{6}\left(1-\frac{3 d_{2}}{5}\right)}\left\|u_{x}\right\|_{L^{2}\left(Q_{t}\right)}^{5 m^{*} / 6}\right) .
$$

Combining this with (4.2) (valid for (2.9), see [9]), (4.5) and (2.10) yields that, for some $\alpha_{k}>0$, we have:

$$
\left\|u_{(t), k}-u_{L(t), k}\right\|_{C\left([0, t] ; L^{2}(0,1)\right)} \leq c^{*}(t)\|\varphi\|_{L^{6 / 5}\left(Q_{t}\right)}+\beta(t) c^{*}(t) e^{\alpha_{k} / t} \rightarrow 0 \text { as } t \rightarrow 0 .
$$

Hence (3.5) holds. This completes the proof.

Concluding remark. A typical alternative lumped control is the point control, which is described by source terms of the form $v(t) \delta\left(x-x_{0}\right)$, where $x_{0}$ is the location. The introduction of controls of this type instead of those in (1.1) does not qualitatively change the controllability results (see, e.g. [15] for the linear case), except for creating a purely technical problem of existence and regularity (which, on the other hand, is of interest of by itself). It seems plausible that our results in one space dimension can be extended to cover this case as well. 


\section{REFERENCES}

[1] Sz. Dolecki, Observation for the one-dimensional heat equation. Stadia Math. 48 (1973) 291-305.

[2] C. Fabre, Uniqueness result for Stokes equations and their consequences in linear and nonlinear problems. ESAIM: Control Optimization and Calculus of Variations 1 (1996) 267-302.

[3] C. Fabre, J.-P. Puel and E. Zuazua, Contrôlabilité approchée de l'équation de la chaleur semi-linéaire. C.R. Acad. Sci. Paris 315 (1992) 807-812.

[4] C. Fabre, J.-P. Puel and E. Zuazua, Approximate controllability for the semilinear heat equation. Proc. Royal Soc. Edinburg 125A (1995) 31-61.

[5] H.O. Fattorini and D.L. Russell, Uniform bounds on biorthogonal functions for real exponentials with an application to the control theory of parabolic equations. Quarterly of Appl. Mathematics (1974) 45-69.

[6] L.A. Fernández and E. Zuazua, Approximate controllability of the semilinear heat equation via optimal control. JOTA (to appear).

[7] A. Fursikov and O. Imanuvilov, Controllability and of evolution equations. Lect. Note Series 34, Res. Inst. Math., GARC, Seoul National University (1996).

[8] A.Y. Khapalov, On unique continuation of the solutions of the parabolic equation from a curve. Control and Cybernetics, Quarterly 25 (1996) 451-463.

[9] A.Y. Khapalov, Some aspects of the asymptotic behavior of the solutions of the semilinear heat equation and approximate controllability. J. Math. Anal. Appl. 194 (1995) 858-882.

[10] O.H. Ladyzhenskaya, V.A. Solonikov and N.N. Ural'ceva, Linear and quasi-linear equations of parabolic type. AMS, Providence, Rhode Island (1968).

[11] J.-L. Lions, Remarques sur la contrôlabilité approchée, in Proc. of "Jornadas Hispano-Francesas sobre Control de Sistemas Distribuidos", University of Málaga, Spain (October 1990).

[12] W.A.J. Luxemburg and J. Korevaar, Entire functions and Müntz-Szász type approximation. Trans. AMS 157 (1971) $23-37$.

[13] V.J. Mizel and T.I. Seidman, Observation and prediction for the heat equation. J. Math. Anal. Appl. 28 (1969) 303-312.

[14] F. Rothe, Global Solutions of Reaction-Diffusion Systems. Lecture Notes in Mathematics No. 1072 (Springer-Verlag, Berlin, 1984).

[15] Y. Sakawa, Controllability for partial differential equations of parabolic type. SIAM J. Cont. 12 (1974) 389-400.

[16] T.I. Seidman, The coefficient map for certain exponential sums. Neder. Akad. Wetemsch. Indag. Math. 48 (1986) $463-478$.

[17] J.-C. Saut and B. Scheurer, Unique continuation for some evolution equations. J. Diff. Equat. 66 (1987) 118-139.

[18] L. Schwartz, Étude des sommes d'exponentielles réelles. Actualités Sci. Indust. No. 959 (Hermann, Paris, 1943).

[19] H.X. Zhou, A note on approximate controllability for semilinear one-dimensional heat equation. Appl. Math. Optim. 8 (1982) $275-285$.

[20] E. Zuazua, Finite dimensional null controllability for the semilinear heat equation. J. Math. Pures Appl. 76 (1997) $237-264$.

[21] E. Zuazua, Approximate controllability for semilinear heat equations with globally Lipschitz nonlinearities. Prep. del Depart. de Matematica Applicada, MA-UCM 1998-035, Universidad Complutense de Madrid (1998). 